\title{
Detection and quantitation of HPV in anogenital and oral tissues and fluids of HIV-positive individuals by real-time PCR
}

\author{
William T Seaman ${ }^{1 *}$, Elizabeth Andrews ${ }^{2,4}$, Marion Couch ${ }^{1}$, Erna Milu Kojic ${ }^{5}$, Susan Cu-Uvin ${ }^{5}$, Allison M Deal ${ }^{4}$, \\ Byrd Quinlavin ${ }^{5}$, Julia Seay ${ }^{5}$, Jennifer Webster-Cyriaque ${ }^{1,2,3}$ \\ From $12^{\text {th }}$ International Conference on Malignancies in AIDS and Other Acquired Immunodeficiencies \\ (ICMAOI)
}

Bethesda, MD, USA. 26-27 April, 2010

Human papillomaviruses (HPV) remain a serious world health problem due to their association with anogenital and oral cancers and warts. While over 100 HPV types have been identified, only a subset is associated with malignancy. HPV16 and 18 are the most common oncogenic types, while HPV6 and 11 are the most common types responsible for anogenital warts. These four types cause up to $90 \%$ of $\mathrm{HPV}$-associated disease. While other quantitative PCR (qPCR) assays can be used to detect oncogenic HPV, there is no single tube assay that distinguishes the most frequent oncogenic types and the most common types found in warts. A qPCR assay was developed that allowed for detection and quantitation of these 4 HPV types. Type-specific primer pairs and TaqMan probes allowed single tube multiplex reactions to be performed. Each HPV type was detected over a range from $2 \mathrm{H} 10^{1}$ to $2 \mathrm{H} 10^{6}$ copies/reaction, providing a reliable method of quantitating type-specific HPV. A Sybr Green-based qPCR assay was developed that utilizes degenerate primers targeting the $\mathrm{E} 1$ region of all HPVs. These assays were run in parallel with PCR/ sequence gold standard on 76 oral cancers from HIVnegative individuals. Cervical and oral washes were collected from 25 HIV-positive women and 90 HIVpositive men, respectively, being screened for anogenital neoplasia. Samples were analyzed using the newly developed assays. Of the 115 samples, $16 \%$ were HPV positive. Cervical washes contained HPV types 44, 67, 35, and 68 and oral specimens contained HPV types 16, 11,
$32,6,55,73$, and 70 . These results indicate that these assays can be used to detect and quantitate HPV in clinical samples obtained by noninvasive measures.

\section{Acknowledgements}

This work was supported in part by NIDCR OHARA 1 U01 Al068636-01. This article has been published as part of Infectious Agents and Cancer Volume 5 Supplement 1, 2010: Proceedings of the $12^{\text {th }}$ International Conference on Malignancies in AIDS and Other Acquired

Immunodeficiencies (ICMAOI). The full contents of the supplement are available online at http://www.biomedcentral.com/1750-9378/5?issue=S1 .

\section{Author details}

'Lineberger Cancer Center, University of North Carolina, Chapel Hill, NC, USA. ${ }^{2}$ Division of Infectious Disease, University of North Carolina, Chapel Hill, NC, USA. ${ }^{3}$ Department of Microbiology and Immunology, University of North Carolina School of Medicine, Chapel Hill, NC, USA. ${ }^{4}$ Department of Dental Ecology, University of North Carolina School of Dentistry, Chapel Hill, NC, USA. ${ }^{5}$ Division of Biology and Medicine, Brown University, Providence, $\mathrm{RI}$, USA.

Published: 11 October 2010

\section{doi:10.1186/1750-9378-5-S1-A17}

Cite this article as: Seaman et al:: Detection and quantitation of HPV in anogenital and oral tissues and fluids of HIV-positive individuals by real-time PCR. Infectious Agents and Cancer 2010 5(Suppl 1):A17. 